\title{
The Detection of Defects in Rolling Bearings Based on the Analysis of Vibroacoustic Signal
}

\author{
Boris Gerike \\ The Federal Research Center of Coal and Coal Chemistry of \\ Siberian Branch of the Russian Academy of Sciences, \\ Institute of Coal \\ Kemerovo, Russian
}

\begin{abstract}
In article the approach to identification of defects roller bearings used in the various parts and components of mining machinery and equipment based on the wavelet transform of the vibro-acoustic signals generated by the various defects arising in the supporting elements of power transmission, transforming and actuators mining equipment. Classification of existing methods of diagnostics of technical condition of rolling bearings is resulted. Advantages and disadvantages of these methods are considered. The model of formation of shock pulses in the event of defects is constructed and the possibility of using wavelet transforms for recognizing the technical state is shown. An example of diagnostics of rolling bearings of the gearbox RKC-400 is considered.
\end{abstract}

Keywords - vibro diagnostic, defect, sliding bearing, defect bearing, mining machine

\section{INTRODUCTION}

Currently, the role of diagnostics of technical condition of mining machines and equipment is constantly growing[1]. The main problems of mechanical malfunctions of mining equipment (imbalance, misalignment, defects of gears, etc.) usually lead to problems in the rolling bearings functioning of the support elements in drive mechanism and executive mechanisms (Fig. 1). a)

Fig.1. Defect of double row bearing: a) wear of the rolling elements of the bearing; $b$ ) wear and tear of raceway

There are the following types of bearing damage primary and secondary $[2,3]$.

Primary damage:

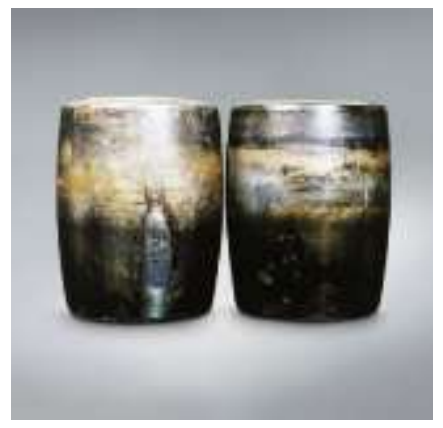

\author{
Andrey Mokrushev* \\ The Federal Research Center of Coal and Coal Chemistry of \\ Siberian Branch of the Russian Academy of Sciences, \\ Institute of Coal \\ Kemerovo, Russian \\ andrey-mokrushev@yandex.ru
}

1) Wear occurs when bearing penetrate debris or insufficient lubrication. It can also be the result of vibrations of non-rotating bearing.

2) Dents on the raceways and rolling elements can occur in cases when the forces are transmitted to the mounting ring via the rolling elements. Equally dents occur when excessively large loads on bearings at a time when the bearings do not rotate. The cause of the dents can be penetration into the bearing of foreign particles.

3) Burrs occur when there is insufficient lubrication of sliding surfaces under load, when there is a transfer of metal particles from one surface to another. The surface look rough. If you encounter scuffing of the bearing material is heated to a temperature at which there is vacation. Occurs local stress concentration that resulted in the formation of cracks and cavities. Also bullies can arise when the rolling elements enter the loaded zone with great acceleration.

4) Surface destruction occur in case of too thin lubricating layer between the tracks and the rolling elements when the top of the roughness briefly touch each other. While on the surface minute cracks occur. In the process of surface destruction of the initially microscopically small, then increase rapidly and, in the end, prevent a smooth rotation of the bearing. Cracks of the described type can speed up the process of formation of fatigue cracks under the surface of the raceways and thereby reduce the durability of the bearing. With adequate lubrication there is no risk of destruction of the described type as long as the lubricating layer becomes too thin, either due to changes in oil viscosity due to temperature increase or excessive increase in workload.

5) Atmospheric corrosion occurs in the case of penetration into the bearing to atmospheric moisture or an aggressive environment in such volume, that the break (dilute) lubricating film in the contact of bodies and raceways.

6) The passage of electric current through the bearing from one ring through the rolling elements to another ring causing bearing damage. In places of transition process similar to electric arc welding. The bearing material may be heated to the melting temperature. In this form the coated areas of different size in which the material is annealed and hardened again, and sometimes even melted. In places where the metal has been melted, there may also be small moons. 
Secondary damage can be attributed:

1) Fatigue sinks - which are the result of normal fatigue damage that occurs at the end of the normal longterm operation of the bearing. This, however, is not the most frequent cause of bearing failure. In most cases, can have causes different from the process of fatigue fracture of the material. If the shell detected at an early stage, when their sizes are not too large, it often becomes possible to identify the cause of the damage, allowing to develop appropriate cautionary measures, and thereby to prevent reinjure. If the shell has reached a certain stage of development, the presence of damage to detect noise and vibrations.

2) Cracks that occur in the rings due to improper Assembly or disassembly of the bearing, too tight fit of the ring on the tapered shaft neck, can also occur due to burrs.

\section{FAULT DIAGNOSIS METHODS}

Vibrodiagnostics solves the following practical tasks of machine and equipment maintenance: Separation of the set of possible technical conditions of the unit into two subsets: serviceable and faulty;

$\checkmark$ The diagnosis, consisting in determining the nature and localization of one or a group of defects, corresponding to the vibrational state of the unit;

$\checkmark \quad$ Detection of a possible defect at an early stage and forecasting its development over time;

$\checkmark$ Absence, as a rule, of assembly and dismantling of equipment and shortening of the time of diagnosis;

$\checkmark$ Reducing the risk of an emergency situation when operating the equipment.

The disadvantages of vibration analysis are:

a. very weak sensitivity;

b. special requirements for mounting the vibration sensor;

c. the dependence of vibration parameters on a large number of factors and the complexity of the vibration signal, due to the presence of a fault, which requires an in-depth knowledge of the methods of correlation and regression analysis and their correct use;

d. the accuracy of diagnosis in most cases depends on the number of smoothed parameters, for example, the number of SPM ratings[6].

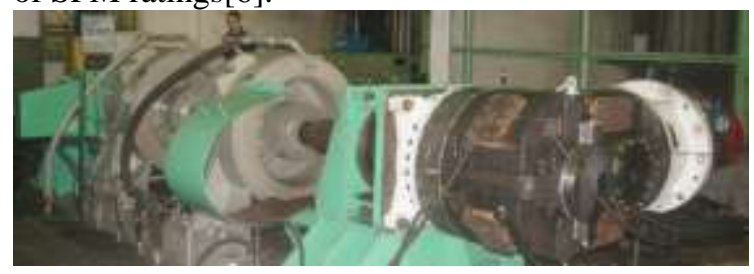

Fig.2. General view of the test stand at JSC "ANGEROMASH»

Complete analysis of the technical condition of the reducer after assembly and run-in on the test bench (Fig.2) will allow not only revealing and correcting manufacturing defects, but also will exclude possibility of delivery on-to the consumer of substandard production[7]. Also, the obtained data can form the basis for the development of an automated quality control system.

\section{RESULTS}

The analysis of vibration control methods leads to the conclusion that it is expedient to apply the method of support masks for automation of control of products intended for the coal industry. This method is based on the fact that the defects that are laid as a result of manufacturing and Assembly work generate vibration in certain frequency bands with a certain ratio of the values of the monitored parameters[7].

Using the method of reference masks to setting the width of the frequency band, its position and values of the criteria evaluation, which are compared the area with the current with the values in arbitrary order. Analyzing the changes of the controlled parameter in the frequency band, the state of the equipment is evaluated and predicted[7-9].

Application of modern technologies of automation of control of technical condition of the equipment allows to carry out an individual approach to each let-out unit at an assessment of its technical condition and establishes threshold values of initial, operational and limit state.
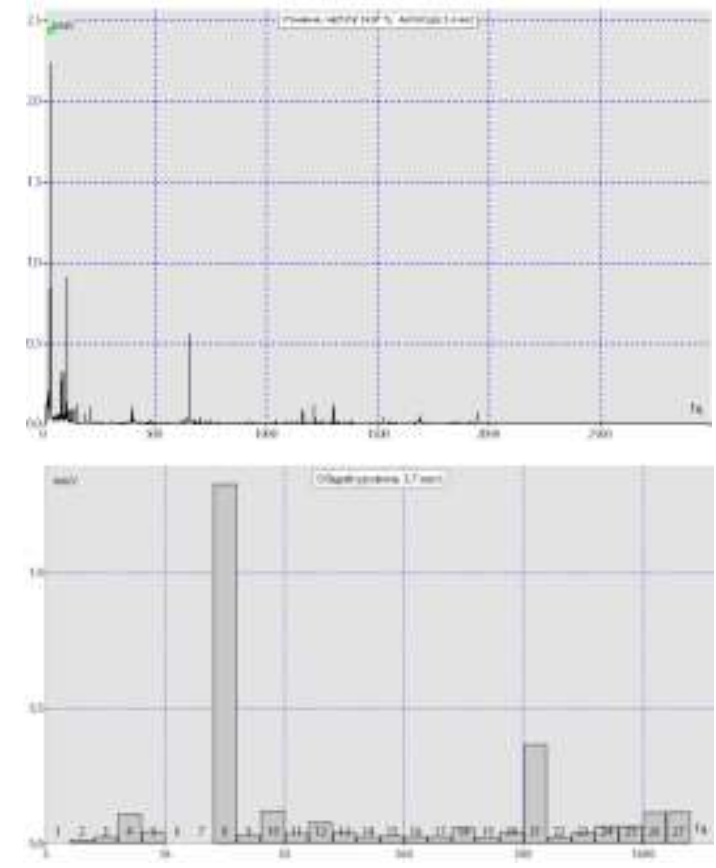

Fig.3. The spectrum of the vibration signal and average spectral mask of the vibration signal

As an example, figure 3 shows a spectrum of vibration signal 1 control point reducer RKC-400 manufactured by JSC "AnzheroMashs" (Fig. 4), and on rice. 3B is its averaged spectral mask. Measurements were made by the vibration analyzer "Corvet", and signal processing on the software platform" Safe Plant "developed by NGO"Diatech" [7].

On the presented example the frequency range (2; $3000 \mathrm{~Hz}$ ) is divided into 27 bands, each of which is normalized according to the RMS value of the vibration velocity VSKZ determined by direct and reverse rotation of the output shaft[7]. 


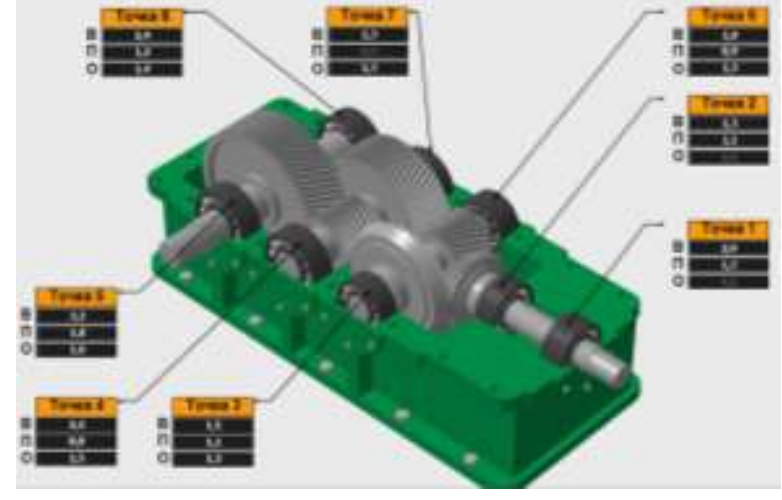

Fig.4. Control points of vibration measurements on gearbox RKC-400

The existing methods of analysis of the technical condition of rolling bearings [7-9] in very rare cases allow to diagnose their failure with sufficient accuracy, since the use of direct spectral analysis of the vibroacoustic signal for the detection of rolling bearing defects is difficult because of the small amplitudes of these frequency components lost in the background of "carpet noise".

The main methods of diagnostics of malfunctions in rolling bearings can be divided into two groups:

- assessment of the status of a rolling bearing is produced on the basis of comparison with data from previous measurements by the method of BEARCON (Schenck), narrow-band spectral analysis, cepstral analysis (Bruel@Kjar), analysis of the kurtosis of a random process (Diamekh 2000), etc [1, 9, 10];

- assessment of the status of a rolling bearing produced according to a single SPM measurement methods (SKF), analyzing envelopes (Boeing), etc.[10].

In order to create a more sensitive method for diagnosing defects, it is necessary to study more deeply the dynamic processes occurring in rolling bearings and their modeling.

So the process of occurrence and action of the shock pulse can be described by the following model:

$$
x(t)=a_{0} \cdot l^{-\beta t} \cdot \operatorname{Cos}\left(\omega \cdot t+\varphi_{0}\right)
$$

Where $x-$ is the offset, $a_{0}-$ is the initial amplitude, $\omega-$ the frequency of the damped oscillations $\beta-$ the speed of the oscillation damping. The frequency of natural oscillations of the system is understood here as the frequency with which the system would oscillate in the absence of resistance forces. The graph of this model is shown in fig. 5. In this model, a one-piece, homogeneous system is considered.

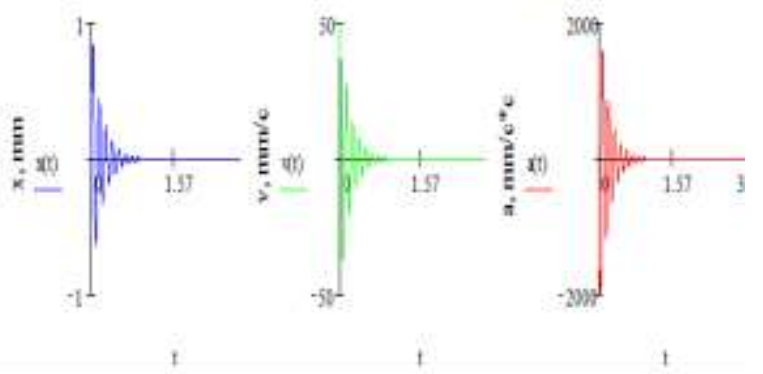

Fig.5. Vibration displacement, vibration speed and vibration acceleration
The action time of the exciting force is very small and is often a fraction or a unit of milliseconds. Each pulse force is from a defect occurring in the bearing elements and the elements of the mechanical design of the unit shall be considered as free fading of the vibrations of the. Since the excitation force is short-term, the frequency spectrum of the resulting free oscillations is very wide and can cover a frequency band from hundreds of Hertz to hundreds of kilohertz.

This explains the successful use of various diagnostic methods for the diagnosis of rolling bearings, analyzing vibrations both in the zone of audible frequencies and in the zone of ultrasound and above[9,10,11].

Fig. 6 shows the shape of the vibration acceleration signal recorded on a non-working experimental setup, on which were held non-strong periodic blows with a metal hammer.

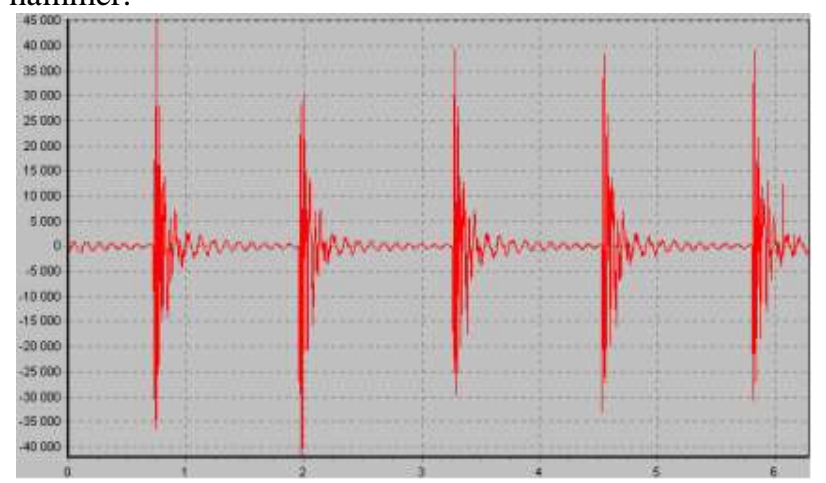

Fig.6. The signal of vibroacceleration

For each frequency is also characterized by different attenuation rate. Fig. 8 shows the change of amplitudes of different frequency components of the Central (located in the middle of the time sweep) shock pulse in time. The slices shown are separated by equal time intervals.

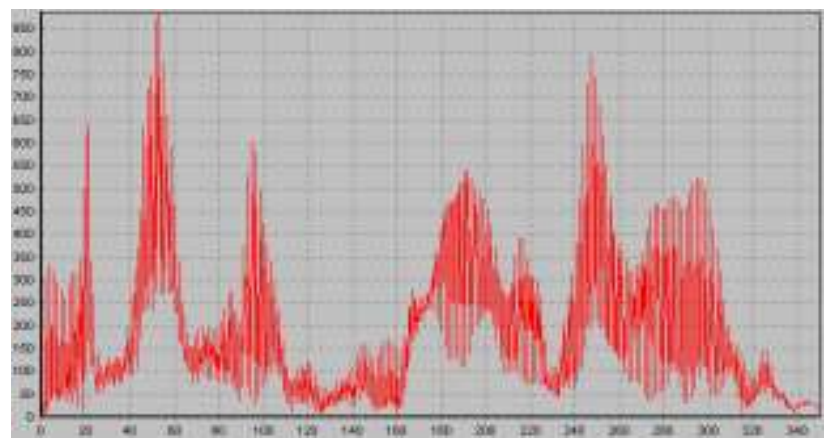

Fig.7. The spectrum of the signal of acceleration

Fig. 8 shows the change of amplitudes of different frequency components of the Central (located in the middle of the time sweep) shock pulse in time. The slices shown are separated by equal time intervals. 


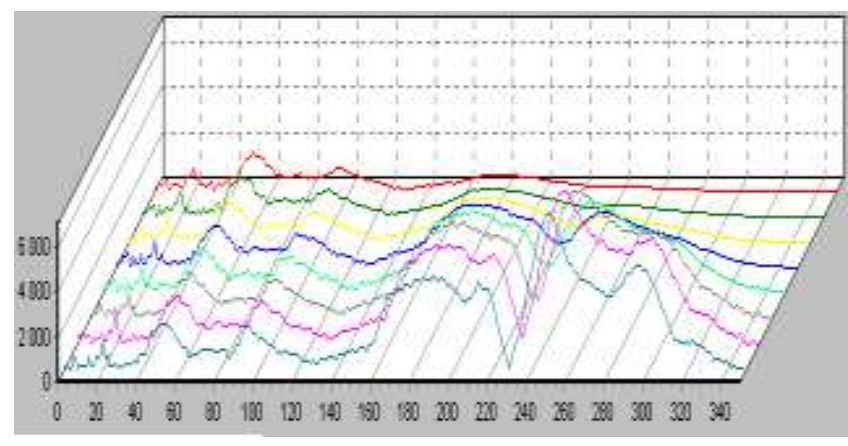

Fig.8. The evolution of the frequency of the pulse in time

In the 70 s of the 20th century appeared, the so-called wavelet methods (methods of bursts). There are two limitations to the wavelet function:

1. it should be quite localized (go to zero with distance from the origin)

2. the integral of the function on $(-\infty ;+\infty)$ must be equal to zero.

$W_{\Psi} f(a, b)=\frac{1}{\sqrt{C_{\Psi}}} \int_{-\infty}^{\infty} \frac{1}{\sqrt{|a|}} \Psi\left(\frac{b-x}{a}\right) f(x) d x$.

where $\Psi$ - wavelet function, $\alpha$-scale, $b$ - shift.

The normalizing factor is equal to

$C_{\Psi}=2 \pi \int_{-\infty}^{\infty} \frac{|\Psi(\omega)|^{2}}{|\omega|} \mathrm{d} \omega<\infty$.

Where $\Psi(\omega)$ - Fourier image of the wavelet $\Psi$.

Freedom in the choice of basic functions $\Psi\left(\frac{b-x}{a}\right)$ allowed to introduce many types of wavelets: Haar, Dobeshi, Gauss, Morlaix, etc.

At its core, the wavelet transform is a representation of a signal in the form of short "bursts" of the same shape, which can be shifted and stretched along the time axis. This is the fundamental difference from the infinite Fourier transform waves[11, 13].

In addition to the continuous wavelet transform discussed above, there is a discrete transformation in which the filtration process takes place. This gives rise to two such concepts as approximation (approximation) and details. Approximation is a high-scale high-frequency components, parts are low-scale high-frequency components. The result is that the original signal is split into two signals that complement each other, giving twice as much data about the original signal. Compared with the decomposition of signals into Fourier series, wavelets have the ability to represent local features of signals with a greater degree of accuracy and solve problems of detecting equipment defects in a more accurate, complex way $[14,15]$.

The development of methods and means of technical diagnostics in relation to the operating conditions of bearing units of mining machines is an important factor in further improving the operation of equipment, ensuring its safety and the possibility of preventing emergency operation modes and sudden failures. 30-60\% of failures of mining equipment occurs due to the failure of bearing units. The increase of the coefficient of the technically sound condition of mining machines is possible due to the introduction of an automated control system. To develop such a system, a software package is developed that will register, process and inform about the actual condition of the bearing unit of the mining machine.

Based on the wavelet transform, a software complex was developed to calculate the characteristic frequencies generated by the interaction of non-existent elements. It is possible to accurately detect bearing defects in the analysis, the characteristic frequencies can accurately identify types of defects. The purpose of the software package is as follows:

1. Real-time monitoring of bearing unit state change.

2. To prevent catastrophic failure of the bearing unit.

3. Plan the repairs of the mining machine.

4. Improve labor safety.

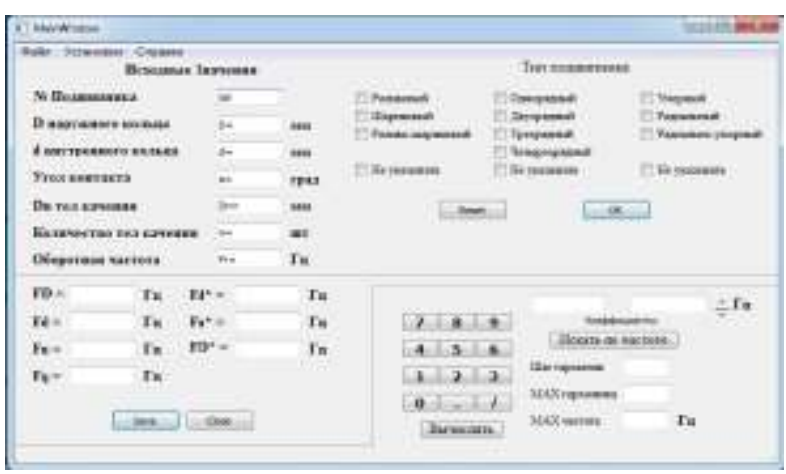

Fig.9. Program interface

How to work with the program

In the Source values area, you must enter data for the calculation:

1. The diameter of the inner ring

2. The diameter of the outer ring

3. Contact angle

4. The diameter of the rolling elements

5. Number of rolling elements

6. Reverse frequency

Then into software values of the spectrum of the vibration signal obtained by the vibration analyser in the measurement of the vibration reducer mining machinery. Select the family of wavelet function further on these values is directly calculated bearing frequencies.

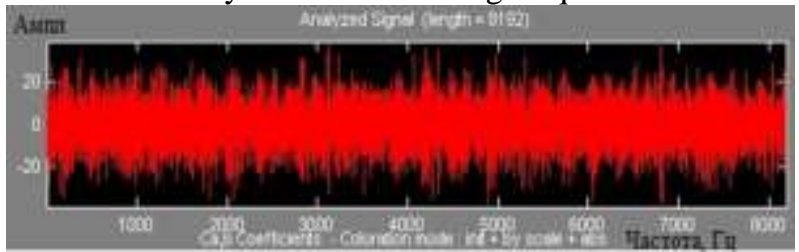

Fig.10. Wavelet transform of the Morlet vibration spectrum

Fig. 10 shows the spectrum of the vibration signal obtained by the software system, the spectrum shows the values of the amplitude characteristic of the wear of the rolling elements and the wear of the racetrack of the rolling bearing. 


\section{CONCLUSIONS}

Using the prognostic model based on the statistical results of vibrodiagnostics, it is possible to accurately estimate the fault under study, as well as to predict the residual life of the unit or unit and to carry out effective planning of repair work, prevent the occurrence of emergency failures. As a result, the proposed solution will minimize the costs associated with the sudden failure of rolling bearings, optimize logistics and warehousing. All conditions will be created for the transition to a qualitatively new system of management of maintenance of mining equipment.

\section{REFERENCES}

[1] Diagnostics of mining machines and equipment. Textbook. B.L. Gerike, P.B. Gerike, V.S.Kvaginidze. Moscow «Nikitskygate», 2012. $400 \mathrm{p}$.

[2] Kelly, S. Graham. Advanced vibration analysis. 2013. 637 p. (Dekker mechanical engineering).

[3] Kostyukov V.N, Naumenko A.P. Fundamentals of vibro-acoustic diagnostics and monitoring

of machines. A tutorial. Omsk. 2011. 368 p.

[4] Salnikov A. F. Vibro-acoustic diagnostics of technical objects. A tutorial. Perm. 2011. 246 p.

[5] Kravchenko V. M., Sidorov V. A., Sedush V. J. Technical diagnostics of mechanical equipment.

The textbook. Donetsk. 2009. 459 p.

[6] Kuzin E. G., Gericke, B. L. condition Monitoring of gearboxes frequency-regulated electric drive of the mine belt conveyors. // Bulletin of the research center for work safety in the coal industry, no 1, 2016. p. $82-88$

[7] Delvecchio, S.; Delia, G.; Mucchi, E.; Dalpiaz, G. Advanced signal processing tools for the vibratory surveillance of assembly faults in diesel engine cold tests, Journal of Vibration and Acoustics, April 2010, Vol. 132, Issue2, 021008-10, ISSN: 1048-9002, doi:10.1115/1.4000807. [8] Zakharov A. Yu., Shiryamov D. V. determination of the critical value of the resistance to the rotation of the conveyor rollers.// Mining equipment and electromechanics, 2016. No. 1. p. 3-8.

[9] State standard R ISO 13373-2-2009. Status monitoring and diagnostics of machines. Vibratory condition monitoring of machines. Part 2. Processing, analysis and presentation of the results of the vibration measurement. M.: STANDARTINFORM, 2009. p. 33.

[10] Nondestructive testing: Handbook: In 8 volumes / Under the General editorship of V. V. Klyuev. Vol. 7: In 2kN. KN. 1: V. I. Ivanov, I. E. Vlasov. The method of acoustic emission. KN. 2: F. Ya.Balitsky, A V. Barkov, N.. Barkov etc. Vibration diagnostics. - 2nd ed. Rev. M.: Mashinostroenie, 2006. 829 p.

[11] One Estimation of the Technical Condition of the Selective Headers./ V. Kovalev, A. Khoreshok, B. Gerike, A. Meshkov. Regarding One Estimation of the Technical Condition of the Selective Headers. //The conferecewas funded by RFBR according to the research project \# 16-05- 20506. October, 10-12, 2016, Kemerovo, Russia. Atlantis Press. Amsterdam, Paris, Beijing. 2016. P.291-296.

[12] Rudloff L., Arghir M., Bonneau O., Guingo S., Chemla G., Renard E. Experimental Analysis of the Dynamic Characteristics of A Hybrid Aerostatic Bearing. Journal of Engineering for Gas Turbines and Power, vol. 134 (18). 2012.

[13] Troubleshooting bearings ./ NSK Motion \& Control, 2009. 42 p.

[14] Damage of rolling bearings and their causes./ SKF AB, 2002. 46 p.

[15] B. L. Gerike, P. B. Gerike. one assessment of residual life of main fans. // Examination of industrial safety in the Siberian Federal district: the collection of proceedings/ under the editorship of Smirnova A. N. T. 2. M.: Mashinostroenie, 2015. P. 85-89. 